\title{
Hábitos alimentares de tubarões-martelo jovens, Sphyrna zygaena (Carcharhiniformes: Sphyrnidae), no litoral sul do Brasil
}

\author{
Hugo Bornatowski ${ }^{1,2,4}$, Luciano Costa ${ }^{3}$, Maurício de Castro Robert ${ }^{3}$ \& Juliana Ventura da Pina ${ }^{3}$ \\ Biota Neotropica $v 7(n 1)$ \\ http://www.biotaneotropica.org.br/v7n1/pt/abstract?short-communication+bn00907012007 \\ Recebido em 20/04/06 \\ Versão reformulada recebida 15/09/06 \\ Publicado em 01/01/07 \\ ${ }^{1}$ Faculdades Integradas Espírita - FACIBEM, Rua Tobias de Macedo Júnior, 333, Santo Inácio, \\ CEP 82010-340, Curitiba, PR, Brasil \\ ${ }^{2}$ Grupo de Pesquisas em Ictiofauna - GPIc, Museu de História Natural Capão da Imbuia, \\ Rua Prof. Benedito Conceição, 407, CEP 82810-080, Curitiba, PR, Brasil, \\ e-mail: anequim.bio@gmail.com \\ ${ }^{3}$ Laboratório de Ictiologia Estuarina, Departamento de Zoologia, Universidade Federal do Paraná, \\ CP 19020, CEP 81531-980, Curitiba, PR, Brasil \\ e-mail:tetragonisca@yahoo.com.br,mauriciorobert@bol.com.br, julianavpina@hotmail.com \\ ${ }^{4}$ Autor para correspondência: Hugo Bornatowski,e-mail: anequim.bio@gmail.com
}

\begin{abstract}
Bornatowski, H., Costa, L., Robert, M.C. \& Pina, J.V. Feeding habits of young smooth hammerhead sharks, Sphyrna zygaena (Carcharhiniformes: Sphyrnidae), in the Southern Coast of Brazil. Biota Neotrop. Jan/Apr 2007 vol. 7, no. $1 \mathrm{http} / /$ www.biotaneotropica.org.br/v7n1/pt/abstract?short-communication+bn00907012007 ISSN 1676-0603.

The present study was conducted in two artisan fishing communities in the cities Guaratuba (Paraná) and Itapoá (Santa Catarina), in the South Brazilian coast. Stomach contents of Sphyrna zygaena specimens were identified, showing three categories of food items. The most important feeding categories were teleosts $(78.6 \%)$ and cephalopods (60.7\%), represented by the sardine Harengula clupeola the squid Loligo sp., both considered the main food items of $S$. zygaena in the studied region.

Keywords: Elasmobranchii, feeding, artisanal fishing communities, Paraná State coast.

\section{Resumo}

Bornatowski, H., Costa, L., Robert, M.C. \& Pina, J.V. Hábitos alimentares de tubarões-martelo jovens, Sphyrna zygaena (Carcharhiniformes: Sphyrnidae), no litoral sul do Brasil. Biota Neotrop. Jan/Apr 2007 vol. 7, no. 1 http://www.biotaneotropica.org.br/v7n1/pt/abstract?short-communication+bn00907012007 ISSN 1676-0603.

O presente estudo foi conduzido em duas comunidades da pesca artesanal nas cidades Guaratuba (Paraná) e Itapoá (Santa Catarina), no sul da costa brasileira. Os conteúdos estomacais de indivíduos Sphyrna zygaena foram identificados, sendo os itens alimentares agrupados em três categorias. As categorias mais importantes foram teleósteos $(78,6 \%)$ e cefalópodes $(60,7 \%)$, representados pela sardinha Harengula clupeola e a lula Loligo sp., ambos considerados como principais itens alimentares de $S$. zygaena na região estudada.
\end{abstract}

Palavras-chave: Elasmobranchii, alimentação, pesca artesanal, costa paranaense. 


\section{Introdução}

A importância dos tubarões é fortemente acentuada do ponto de vista trófico, já que, consumindo grande espectro de organismos, a maioria das espécies ocupa posição de destaque na cadeia alimentar dos ecossistemas marinhos, sobretudo daqueles de regiões tropicais e subtropicais (Camhi et al. 1998). Sphyrna zygaena é uma espécie de tubarão pelágico costeiro ou semioceânico e distribui-se desde a superfície até $150 \mathrm{~m}$ de profundidade, apresentando uma ampla distribuição sobre a plataforma continental de todos os continentes (Compagno 1984, Steel 1985, Gadig 2001). No Brasil, aparentemente é mais comum nas regiões Sudeste e Sul, onde exemplares jovens podem ser encontrados próximos da costa nos meses de inverno e os adultos são capturados pelas frotas espinheleiras na área oceânica, onde, juntamente com Sphyrna lewini, representa a grande maioria dos tubarões martelos capturados (Gadig 2001). Sphyrna zygaena é muito comum nas pescas artesanais da região paranaense, onde é representado geralmente por neonatos e jovens (Barletta \& Corrêa 1989, Charvet 1995a,b, Costa \& Chaves 2002).

No Brasil, trabalhos sobre alimentação de tubarões costeiros ainda são escassos (Vaske Jr. et al. 1993, Capitoli et al. 1995, Muto et al. 1995, Lessa et al. 1999, Vaske Jr. \& Rincón-Filho 1998, Lima et al. 2000). Desta forma o presente estudo traz novas informações sobre os hábitos alimentares de jovens de $S$. zygaena na costa paranaense, identificando a composição dos itens alimentares consumidos.

\section{Materiais e Métodos}

Entre julho de 2001 e março de 2003 e em maio de 2004 foram realizadas visitas nas comunidades pesqueiras artesanais de Brejatuba ( $25^{\circ} 53^{\prime} \mathrm{S}$ e $\left.48^{\circ} 33^{\prime} \mathrm{W}\right)$, município de Guaratuba (PR), e Barra do Saí ( $26^{\circ} 00^{\prime} \mathrm{S}$ e $\left.48^{\circ} 36^{\prime} \mathrm{W}\right)$, município de Itapoá (SC). Em cada ocasião os desembarques diários, que ocorreram em geral entre 9 e 14 horas, foram observados em relação à presença de indivíduos de S. zygaena. Dentre todas as modalidades de pesca praticadas na região, a captura de $S$. zygaena apenas foi observada nos fundeios, pescaria realizada com rede de emalhe fixa ao fundo numa profundidade máxima de 20 m (Robert 2004).

No campo foram tomados os dados de comprimento total (CT), com o auxílio de uma trena, e de peso total (PT), com o auxílio de uma balança do tipo dinamômetro. Os estágios de desenvolvimento reprodutivo foram determinados de acordo com Castro (1983), Hazin et al. (2001), Motta (2001) e Andrade (2004). Desta forma, os recém-nascidos foram reconhecidos por apresentarem cicatriz umbilical aberta. Foram considerados jovens fêmeas que não demonstravam, macroscopicamente, atividade vitelogênica (ovários com coloração esbranquiçada) e machos com clásperes descalcificados ou não totalmente calcificados. A calcificação dos clásperes foi analisada manualmente, observando a flexibilidade. O sistema digestório foi removido e fixado em solução de formol a 4\%. Após a biometria realizada, os indivíduos foram devolvidos aos pescadores para serem comercializados.

No laboratório os conteúdos estomacais foram examinados com auxílio de um microscópio estereoscópico. Os itens foram identificados até o menor nível taxonômico possível com auxílio de publicações especializadas, mas devido à impossibilidade de sempre se discriminar estes itens em um menor nível taxonômico, os mesmos foram agrupados em grandes grupos taxonômicos.

Para a análise da importância dos itens alimentares foi utilizado o método de freqüência de ocorrência (FO), que avalia o percentual de estômagos em que determinado item ocorre e o método de número de pontos $(\mathrm{P})$, onde a contribuição de cada item é determinada pela proporção de quadrículas ocupadas pelo item em uma superfície plana quadriculada em relação ao número total de quadrículas ocupadas pelo conteúdo (Hynes 1950, Hyslop 1980). Para análise da importância efetiva de cada item na alimentação da espécie foi utilizado o Índice Alimentar (IAi), segundo uma adaptação do método de Kawakami \& Vazzoler (1980) proposta por Chaves \& Vendel (1996). Os bicos isolados de cefalópodes nos estômagos foram contabilizados apenas para cálculos de frequiência de ocorrência, com a finalidade de evitar superestimativas nos cálculos do IAi (Vaske-Jr. \& Castello 1998).

\section{Resultados}

Foram examinados 30 exemplares jovens de tubarões martelo durante o período de estudo. A amplitude de tamanho dos indivíduos variou entre 78 e $133 \mathrm{~cm}$, e o peso, entre 1565,90 e 8993,34 g. A proporção sexual machos/fêmeas encontrada foi de 1:1 $(\mathrm{gl}=1$; $\left.\chi^{2}=0,12\right)$.

A análise de 30 estômagos, todos apresentando algum conteúdo, revelou uma pequena variedade de itens (Tabela 1). Teleostei foi o

Tabela 1. Itens alimentares encontrados nos conteúdos estomacais de 30 indivíduos de Sphyrna zygaena analisados. *Parasitos registrados no trato digestivo; não fazem parte da dieta do animal.

Table 1. Feeding items found in the stomach contents of 30 analyzed individuals of Sphyrna zygaena. *Parasites registered in the digestive treatment; they are not part of the specimens' diet.

\begin{tabular}{l}
\hline \\
Filo Arthropoda \\
Classe Crustacea \\
Ordem Isopoda \\
Ordem Decapoda \\
Família Portunidae \\
Callinectes sp.
\end{tabular}

\section{Filo Mollusca}

\section{Classe Cephalopoda}

Ordem Teuthoidea

Família Loligindae

Loligo sp.

\begin{tabular}{l}
\hline Filo Chordata \\
Divisão Teleostei \\
Ordem Clupeiformes \\
Família Clupeidae \\
Harengula clupeola \\
Ordem Beloniformes \\
Família Hemiramphidae \\
Ordem Perciformes \\
Família Haemulidae \\
Família Carangidae \\
Trachinotus carolinus \\
Família Trichiuridae \\
Trichiurus lepturus \\
Família Sciaenidae \\
\hline Teleósteos não Identificados \\
\hline Matéria Digerida \\
\hline Filo Nematoda* \\
\hline Classe Secernentea \\
Ordem Ascaridida \\
Família Anisakidae
\end{tabular}


grupo que apresentou a maior diversidade de presas ingeridas por S. zygaena (Tabela 1). Com relação à importância alimentar, teleósteos, cefalópodes e crustáceos foram os grupos que se destacaram (Tabela 2), sendo representados principalmente pela sardinha-cascuda Harengula clupeola, pela lula Loligo sp. e pelo siri Callinectes sp. É importante apontar o elevado percentual de ocorrência de vermes nematóides da família Anisakidae nos estômagos dos tubarões estudados (Tabela 2). Também foram encontrados alguns objetos (pedaços de plásticos e escova) dentro dos estômagos.

\section{Discussão}

Sphyrna zygaena apresentou hábito teutófago e ictiófago durante sua fase juvenil. A lula do gênero Loligo sp. foi encontrada na maioria dos estômagos, podendo ser considerada como fonte alimentar de extrema importância para essa espécie de tubarão, porém sua importância provavelmente foi subestimada, já que a musculatura deste cefalópode é provavelmente de digestão mais rápida e o volume encontrado nos estômagos é composto por bicos destes animais. O acúmulo de bicos nos estômagos é fato conhecido em algumas espécies de peixes (Zavala-Camin 1981, Mello 1992, Santos 1992, Vaske-Jr. 1992). Vaske-Jr. \& Ríncon-Filho (1998) aconselham desconsiderar bicos isolados em cálculos de quantificação numérica, no intuito de evitar superestimativas.

O estudo realizado por Cortês (1999) revelou que $S$. zygaena tem preferência por cefalópodes seguidos de teleósteos, resultado este não confirmado no presente trabalho, onde S. zygaena alimentou-se primeiramente de teleósteos. Outros estudos realizados com jovens de S. lewini na costa sudeste do Brasil (Namora et al. 2000) e na costa de Kane'ohe Bay, Hawai (Bush 2003), revelaram que essa espécie de tubarão martelo tem preferência alimentar por peixes teleósteos e crustáceos, com baixa ocorrência de cefalópodes. Sphyrna tiburo foi estudado no sudeste da Flórida por Cortês et al. (1996) e demonstrou grande predominância de crustáceos, principalmente o siri-azul Callinectes sapidus, em sua dieta. As diferenças observadas podem então estar relacionadas com a disponibilidade dos itens no ambiente, conforme já sugerido por Wootton (1990), Moyle \& Cech (1982) e Weatherley (1972), muito embora também existam variações em função de aspectos comportamentais (Zavala-Camin 1996).

De acordo com os itens registrados, a alimentação de $S$. zygaena baseou-se em organismos pelágicos. Harengula clupeola foi o peixe com maior número representativo entre os teleósteos. Essa espécie vive em cardumes pequenos e é comum nas regiões costeiras do estado do Paraná (Spach et al. 2003, Godefroid et al. 2003, Fávaro 2004, Pichler 2005). Esses mesmos autores ainda relataram a grande ocorrência de Clupeidae, Hemiramphidae, Carangidae, Trichiuridae e Scianidae na costa paranaense. As lulas do gênero Loligo são nec-

Tabela 2. Frequiência de ocorrência (FO), pontos (P) e Índice de Importância Alimentar (IAi) dos itens registrados nos estômagos de 30 exemplares de Sphyrna zygaena. *Itens não contabilizados no cálculo do IAi.

Table 2. Frequency of occurrence (FO), points (P) and Index of Alimentary Importance (IAi) of items registered in the stomachs of 30 units of Sphyrna zygaena. *Items not entered for the IAi calculation.

\begin{tabular}{lccc}
\hline \multicolumn{1}{c}{ Itens } & FO $(\boldsymbol{\%})$ & P $(\%)$ & IAi $(\%)$ \\
\hline Teleósteos & 78,57 & 47,77 & 70,10 \\
Cefalópodes & 60,71 & 22,63 & 29,89 \\
Crustáceos & 3,57 & 0,13 & 0,01 \\
Matéria Digerida* & 85,71 & 28,65 & - \\
Nematódeos* & 48,64 & - & - \\
Objetos* & 10,71 & 0,09 & - \\
\hline
\end{tabular}

tônicas e representam cerca de $80 \%$ dos cefalópodes ocorrentes no sul do Brasil (Haimovici \& Perez 1991, Haimovici 1997 e Cergole 1999). Na costa paranaense a abundância de Loligo sp. também foi registrada na alimentação de Synodus foetens (Kagiwara \& Abilhoa 2000). Desta forma, podemos dizer que peixes ósseos, cefalópodes e crustáceos compõem uma porção importante da dieta desta espécie de tubarão, porém os motivos que explicariam a maior representatividade de uma ou outra categoria alimentar ficam condicionados à realização de análises mais robustas, tanto das condições ambientais locais quanto das interações que determinam a composição e estrutura destas comunidades de presas.

A presença de vermes anisakídeos nos estômagos de $S$. zygaena pode estar associada à ingestão de peixes e lulas contaminados (Aragort 2003) e representa uma ocorrência ocasional, assim como o registro de pedaços de plásticos e uma escova. Neste caso, tais ocorrências parecem demonstrar o hábito oportunista dos tubarões, visto que vários estudos prévios apontam a ocorrência de objetos dessa natureza (Hazin et al. 1994, Vaske-Jr. \& Ríncon-Filho 1998, Simpfendorfer et al. 2001, McCord \& Campana 2003).

\section{Agradecimentos}

Agradecemos aos pescadores da Barra do Saí e Brejatuba, por permitirem que o trabalho fosse realizado, e Paulo T. C. Chaves, por disponibilizar o espaço físico e os equipamentos do Laboratório de Ictiologia Estuarina.

\section{Referências Bibliográficas}

ANDRADE, A.C. 2004. Aspectos da reprodução e estrutura populacional de duas espécies de caçonete, Rhizoprionodon lalandii (Valenciennes, 1839) e Rhizoprionodon porosus (Poey, 1861), capturadas pela frota artesanal da Associação de Pescadores do Recreio dos Bandeirantes, no município do Rio de Janeiro, Brasil. Monografia. Universidade Rural do Rio de Janeiro, Rio de Janeiro.

ARAGORT, W. 2003. Parásitos anisákidos en peces de interés comercial y su riesgo para la salud. In CENIAP HOY No. 2, mayo-agosto 2003. ISSN: 1690-4117. Maracay, Aragua, Venezurela. URL: http://www.ceniap.gov. ve/ceniaphoy/articulos/n2/texto/waragort.htm. Visitado em 12/03/2006.

BARLETTA, M. \& CORRÊA, M.F.M. 1989. Chondrofauna do Complexo Estuarino da Baía de Paranaguá e adjacências, PR. Levantamento e produtividade pesqueira. Resumos. In IV Reunião do Grupo de Trabalho sobre Pesca e Pesquisa de Tubarões e Raias no Brasil. Universidade Federal de Pernambuco, Tamandaré, p.2.

BUSH, A. 2003. Diet and diel feeding periodicity of juvenile scalloped hammerhead sharks, Sphyrna lewini, in Kane'ohe Bay, Oahu, Hawaii. Environ. Biol. Fishes 67:1-11.

CAMHI, M., FOWLER, S., MUSICK, J., BRÄUTIGAN \& FORDHAN, S. 1998. Sharks and their relatives. Ecology and Conservation. Occas. Pap. IUCN Spec. Surv. Com. 20:1-39.

CAPITOLI, R.R., RUFFINO, M.L. \& VOOREN, C.M. 1995. Alimentação do tubarão Mustelus schmitti (Springer 1940) na plataforma costeira do estado do Rio Grande do Sul, Brasil. Atlântica 17:109-122.

CASTRO, J.L. 1983. Sharks of the North American Waters. 1 ed. Texas A \& M University Press.

CERGOLE, M.C. 1999. Avaliação a ações prioritárias para a conservação de biodiversidade da zona costeira e marinha. nécton - pequenos pelágicos. Disponível em http://www.bdt.fat.org.br/workshop/costa/peqpelagicos. Visitado em 03/06/2006.

CHARVET, P. 1995a. Dados preliminares do levantamento da chondrofauna do litoral do Estado do Paraná. Resumos. In VII Encontro do grupo de trabalho sobre pesca e pesquisa de tubarões e raias no Brasil, Rio Grande, p.27.

CHARVET, P. 1995b. Pesca de elasmobrânquios juvenis no litoral do Estado do Paraná: uma realidade. Resumos. In VII Encontro do grupo de trabalho sobre pesca e pesquisa de tubarões e raias no Brasil, Rio Grande, p.29. 
CHAVES, P.T.C. \& VENDEL, A.L. 1996. Aspectos da alimentação de Genidens genidens (Valenciennes 1839) (Siluriformes Ariidae) na Baía de Guaratuba, Paraná, Brasil. Revta. Bras. Zool. 13(3):669-675.

COMPAGNO, L.J.V. 1984. FAO species catalogue. Sharks of the world: an annotated and illustrated catalogue of shark species known to date. Part 2. Carcharhiniformes.. FAO Fish. Synop. 4:251-655.

CORTES, E., MANIERI, C.A. \& HUETER, R.E. 1996. Diet, feeding habitats, and field feeding chronology of the bonnethead shark, Sphyrna tiburo, in southwest Florida. Bull. Mar. Sci. 58:353-367.

CORTES, E. 1999. Standardized diet compositions and trophic levels of sharks. ICES J. Mar. Sci. 56:707-717.

COSTA, L. \& CHAVES, P.T.C. 2002. Hábitos alimentares, reprodutivos e a importância comercial dos elasmobrânquios para a pesca artesanal no litoral sul do Paraná. Resumos. In X Evento de Iniciação Científica da UFPR, Curitiba, p. 103.

FÁVARO, L.F. 2004. A ictiofauna de áreas do complexo estuarino baía de Paranaguá, Brasil. Tese de Doutorado, Universidade Federal de São Carlos, São Paulo.

GADIG, O.B.F. 2001. Tubarões da costa brasileira. Tese de Doutorado, Universidade Estadual Paulista, Rio Claro.

GODEFROID, R.S., SPACH, H.L., SHWARZ-JR., P. \& QUEIROZ, G.M. 2003. A fauna de peixes da praia do balneário Atami, Paraná, Brasil. Atlântica 25(2):147-161.

HAIMOVICI, M. \& PEREZ, J.A.A. 1991. Abundância e distribuição de cefalópodes em cruzeiros de prospecção pesqueira demersal na plataforma externa e talude continental do sul do Brasil. Atlântica 13(1):189-200.

HAIMOVICI, M. 1997. Cephalopods. In Subtropical convergence environments: the coast and sea in the southwest Atlantic (U. SEELIGER, C. ODEBRECHT \& J.P. CASTELLO, eds.). Springer-Verlag, Heidelberg, p.308.

HAZIN, F.H.N., LESSA, R.P.T. \& CHAMMAS, M. 1994. First observations on stomach contents of the blue shark, Prionace glauca, from southwestern equatorial Atlantic. Rev. Bras. Biol. 54(2):195-198.

HAZIN, F.H.V., FISCHER, A. \& BROADHURST, M.K. 2001. Aspects of reproductive biology of the scalloped hammerhead shark, Sphyrna lewini, of northeastern Brazil. Environ. Biol. Fishes 61:151-159.

HYNES, H.B.N. 1950. The food of fresh-water sticklebacks (Gasterosteus aculeatus and Pygosteus pungitius), with a review of methods used in studies of the food of fishes. J. Anim. Ecol. 19:36-57.

HYSLOP, E.J. 1980. Stomach contents analysis: a review of methods and their application. J. Fish Biol. 17:411-429.

KAGIWARA, F. \& ABILHOA, V. 2000. A alimentação do peixe-lagarto Synodus foetens (Linnaeus, 1766) em um banco areno-lodoso na Ilha do Mel, Paraná, Brasil. Arq. Ciên. Veter. Zoo. UNIPAR 3(1):9-17.

KAWAKAMI, E. \& VAZZOLER, G. 1980. Método gráfico e estimativo de índice alimentar aplicado no estudo de alimentação de peixes. Bol. Inst. Oceanog. 29(2):205-207.

LESSA, R., SANTANA, F.M., RINCON, G., GADIG, O.B.F. \& EL-DEIR, A.C.A. 1999. Avaliação a ações prioritárias para a conservação de biodiversidade da zona costeira e marinha. Biodiversidade de elasmobrânquios do Brasil. Disponível em http://www.bdt.org.br/workshop/costa/elasmo. Visitado em 24/02/2006.

LIMA, G.H.L, DAROS, F.A., MAZZOLENI, R. \& HOSTIM-SILVA, M. 2000 Aspectos da alimentação natural do cação-frango Rhizoprionodon lalandii (Valenciennes, 1841) (Elasmobranchii, Carcharhinidae) no município de Barra Velha, Santa Catarina. Notas Téc. FACIMAR 4:91-96.

McCORD, M.E. \& CAMPANA, S. 2003. A quantitative assessment of the diet of the blue shark (Prionace glauca) of Nova Scotia, Canada. J. Northw. Ad. Fish. Sci. 32:57-63.

MELLO, R.M. 1992. Análise dos conteúdos estomacais, intensidade de alimentação, idade e crescimento do espadarte Xiphias gladius (Xiphioidei: Xiphiidae) no sul do Brasil. Dissertação de Mestrado, Universidade do Rio Grande, Rio Grande.

MOTTA, F.S. 2001. A pesca artesanal e a reprodução de Rhizoprionodon lalandii (Elasmobranchii - Carcharhinidae) no litoral sul do estado de São Paulo. Dissertação de Mestrado, Universidade Estadual Paulista, Rio Claro.

MOYLE, P.B. \& CECH JR., J.J. 1982. Fishes: an introduction to ichthyology. Prentice-Hall, Englewood Cliffs.
MUTO, E.Y., SOARES, L.S.H. \& GOITEIN, R. 1995. Alimentação das raias Raja agassizi e Psammobatis glansdssimilis (Chondrichthyes: Rajidae) da Região Costeira de Ubatuba (SP). Resumos. In XI Encontro Brasileiro de Ictiologia, Campinas, p.11.

NAMORA, R.C., MOTTA, F.S. \& GADIG, O.B.F. 2000. Alimentação de neonatos e jovens de tubarão martelo, Sphyrna lewini, no litoral sul de São Paulo. Resumos. In II Reunião da Sociedade Brasileira para Estudo dos Elasmobrânquios, Santos, p.6.

PICHLER, H.A. 2005. Ictiofauna em planícies de maré da baía dos Pinheiros, Paraná. Dissertação de Mestrado, Universidade Federal do Paraná, Curitiba.

ROBERT, M.C. 2004. Análise da pesca artesanal de fundeio realizada pelas comunidades de Brejatuba, litoral sul do Paraná e da Barra do Saí, litoral norte de Santa Catarina, Brasil. Dissertação de Mestrado, Universidade Federal do Paraná, Curitiba.

SANTOS, R.A. 1992. Relações tróficas do calamar argentino Illex argentinus (Castellanos, 1960) (Teuthoidea: Ommastrephidae), no sul do Brasil. Dissertação de Mestrado, Universidade do Rio Grande, Rio Grande.

SIMPFENDORFER, C.A., GOODREID, A.B. \& McAULEY, R.B. 2001. Size, sex and geographic variation in the tiger shark, Galeocerdo cuvier, from Western Australia waters. Environ. Biol. Fishes 61:37-46.

SPACH, H.L., SANTOS, C. \& GODEFROID, R.S. 2003. Planícies temporais na assembléia de peixes na gamboa do Sucuruí, Baía de Paranaguá, Brasil. Revta. Bras. Zool. 20(4):591-600.

STEEL, R. 1985. Sharks of the world. Blandford Press, New York.

VASKE-JR., T. 1992. Idade, crescimento e alimentação da albacora de lage, Thunnus albacares (Bonaterre, 1788) (Perciformes: Scombridae) explorada no sul do Brasil. Dissertação de Mestrado, Universidade do Rio Grande, Rio Grande.

VASKE-JR, T., HAZIN, F.H.V., EL-DEIR, A.A.C., BEZERRA-JR, J.L. \& BOECKMANN, C.E.V. 1993. Análise do conteúdo estomacal dos principais tubarões pelágicos capturados com espinhel pelágico no Atlântico Sudoeste Equatorial. Resumos. In. VI Reunião do Grupo de Trabalho sobre Pesca e Pesquisa de Tubarões e Raias no Brasil. Recife, PE, p. 37.

VASKE-JR., T. \& RÍNCON-FILHO, G. 1998. Conteúdo estomacal dos tubarões azul (Prionace glauca) e anequim (Isurus oxyrinchus) em águas oceânicas no sul do Brasil. Rev. Bras. Biol. 58(3):445-452.

VASKE-JR., T. \& CASTELLO, J.P. 1998. Conteúdo estomacal da albacoralaje, Thunnus abacares, durante o inverno e primavera no sul do Brasil. Rev. Bras. Biol. 58(4):639-647.

WEATHERLEY, A.H. 1972. Growth and ecology of fish populations. Academic Press, London.

WOOTTON, R.J. 1990. Ecology of teleost fishes. Chapman \& Hall, London.

ZAVALA-CAMIN, L.A. 1981. Hábitos alimentares e distribuição dos atuns e afins (Osteichthyes: Teleostei) e suas relações ecológicas com outras espécies pelágicas das regiões sudeste e sul do Brasil. Tese de Doutorado, Universidade de São Paulo, São Paulo.

ZAVALA-CAMIN, L.A. 1996. Introdução aos estudos sobre alimentação natural em peixes. EDUEM, Maringá.

Título: Hábitos alimentares de tubarões-martelo jovens, Sphyrna zygaena (Carcharhiniformes: Sphyrnidae), no litoral sul do Brasil.

Autores: Bornatowski, H, Costa, L, Robert, MC, Pina, JV

Biota Neotropica, Vol.7 (número 1): 2007

http://www.biotaneotropica.org.br/v7n1/pt/abstract?shortcommunication+bn00907012007

Recebido em 20/04/06 - Versão reformulada recebida 15/09/06 - Publicado em 01/01/07

ISSN 1676-0603 